Global structure of periodicity hubs in Lyapunov phase diagrams of dissipative flows

Vitolo, Renato and Glendinning, Paul and Gallas, Jason A.C.

2011

MIMS EPrint: 2011.57

Manchester Institute for Mathematical Sciences

School of Mathematics

The University of Manchester

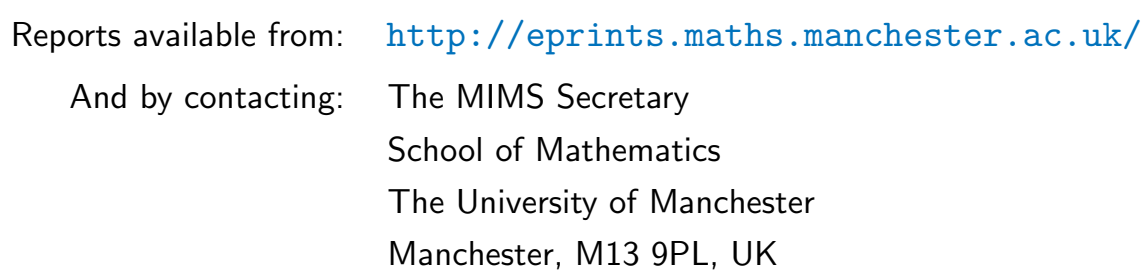

ISSN 1749-9097 


\title{
Global structure of periodicity hubs in Lyapunov phase diagrams of dissipative flows
}

\author{
Renato Vitolo, ${ }^{1}$ Paul Glendinning, ${ }^{2}$ and Jason A.C. Gallas ${ }^{1,3,4}$ \\ ${ }^{1}$ School of Engineering, Computing and Mathematics, University of Exeter, Exeter EX4 $4 Q F$, UK \\ ${ }^{2}$ School of Mathematics, University of Manchester, Oxford Road, Manchester M13 9PL, UK \\ ${ }^{3}$ Departamento de Física, Universidade Federal da Paraíba, 58051-970 João Pessoa, Brazil \\ ${ }^{4}$ Institute for Multiscale Simulation, Friedrich-Alexander Universität, 91052 Erlangen, Germany
}

(Dated: 29 de Maio de 2011, às 20:35)

\begin{abstract}
Infinite cascades of periodicity hubs were predicted and very recently observed experimentally to organize stable oscillations of some dissipative flows. Here we describe the global mechanism underlying the genesis and organization of networks of periodicity hubs in control parameter space of a simple prototypical flow. We show that spirals associated with periodicity hubs emerge/accumulate at the folding of certain fractal-like sheaves of Shilnikov homoclinic bifurcations of a common saddle-focus equilibrium. The specific organization of hub networks is found to depend strongly on the interaction between the homoclinic orbits and the global structure of the underlying attractor.
\end{abstract}

\section{INTRODUCTION}

To study the response of a system with respect to changes in the processes involved is a basic problem in physics and the quantitative sciences in general. For dissipative systems, the problem is formulated in terms of the attractors typically underlying the long-term dynamical behavior, see e.g. [1, 2]. Here one aims to understand the transitions (bifurcations) of the attractors under variation of the system's control parameters.

Numerical computation of Lyapunov exponents provides a convenient tool to classify attractors and bifurcations of dynamical systems. A positive or zero maximal Lyapunov exponent usually indicates sensitivity with respect to the initial conditions or, respectively, temporally regular behavior (e.g. periodic or quasi-periodic) [1, 2]. With the development of fast throughput numerical experiments, it becomes feasible to explore large ranges of parameter space, classifying the system's attractors by their Lyapunov exponents. Lyapunov phase diagrams are graphical summaries of these explorations, describing the attractor type as a function of the control parameters through color or gray scale codes. Such diagrams have been obtained both numerically [3-16] and experimentally [17] and may offer a wealth of information on the the nature of the attractors and their bifurcations. Lyapunov phase diagrams are useful since they code only the stable dynamics and, hence, the dynamics which is likely to be observable and relevant in any physical system.

A specific class of spiral-like structures has been identified in Lyapunov phase diagrams of dissipative flows as diverse as piecewise-linear resistive electric circuits, electrical circuits with smooth nonlinearities, certain lasers, chemical oscillators and other paradigmatic flows [3$7,15,16]$. These structures consist of a double alternation of nested spirals converging to a central point: the so-called periodicity hub. Fig. 1 shows a periodicity hub in the $(c, a)$-parameter plane of Rössler's oscillator [18]:

$$
\dot{x}=-y-z, \quad \dot{y}=x+a y, \quad \dot{z}=(b+z) x-c z,
$$

where $b=0.3$. Two groups of nested spiral-shaped re-

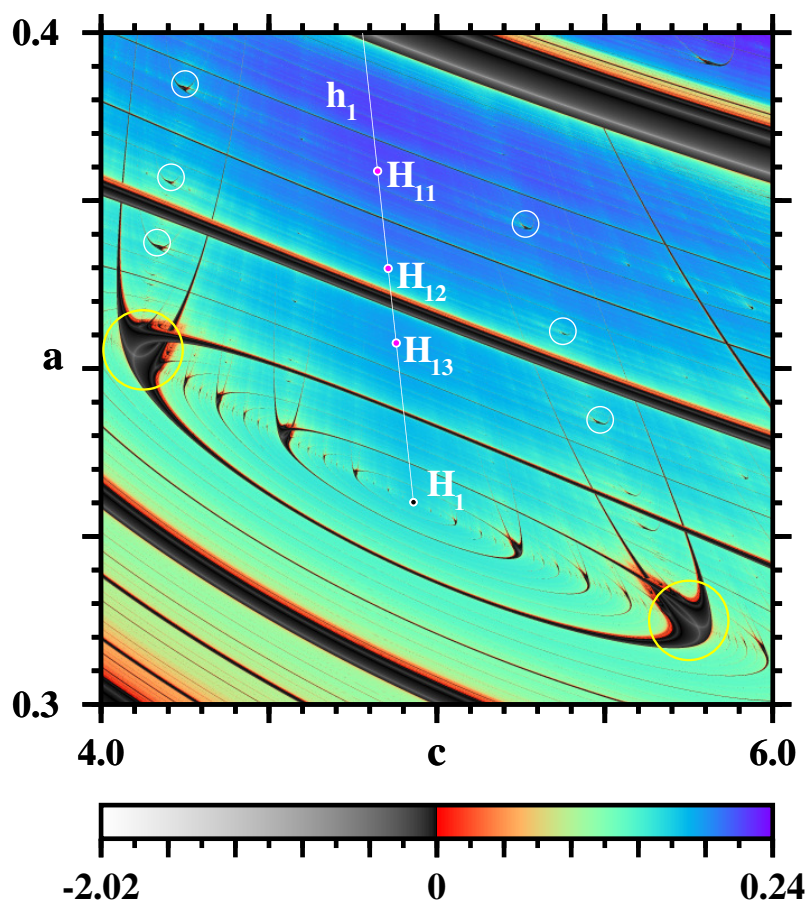

FIG. 1. (Color online) Lyapunov phase diagram for Rössler's oscillator, with four dots marking periodicity hubs $H_{1}$ and $H_{1 j}, j=1,2,3$ and with a curve $h_{1}$ of Shilnikov homoclinic bifurcations. Grey tones, identifying the periodicity spirals, are proportional to the maximum nonzero Lyapunov exponent. Color, identifying the chaoticity spiral, depends on the maximum Lyapunov exponent, which is positive. The diagram displays $2400^{2}=5.76 \times 10^{6}$ parameter points.

gions accumulate in the parameter plane around the periodicity hub $H_{1}$ : periodicity and chaoticity spirals. Individual periodicity (chaoticity) spirals are characterized by a zero (positive) maximum Lyapunov exponent, corresponding to periodic (chaotic) stable oscillations in phase space. Both groups seems to contain infinitely many spirals. As parameters approach $H_{1}$ within a single period- 

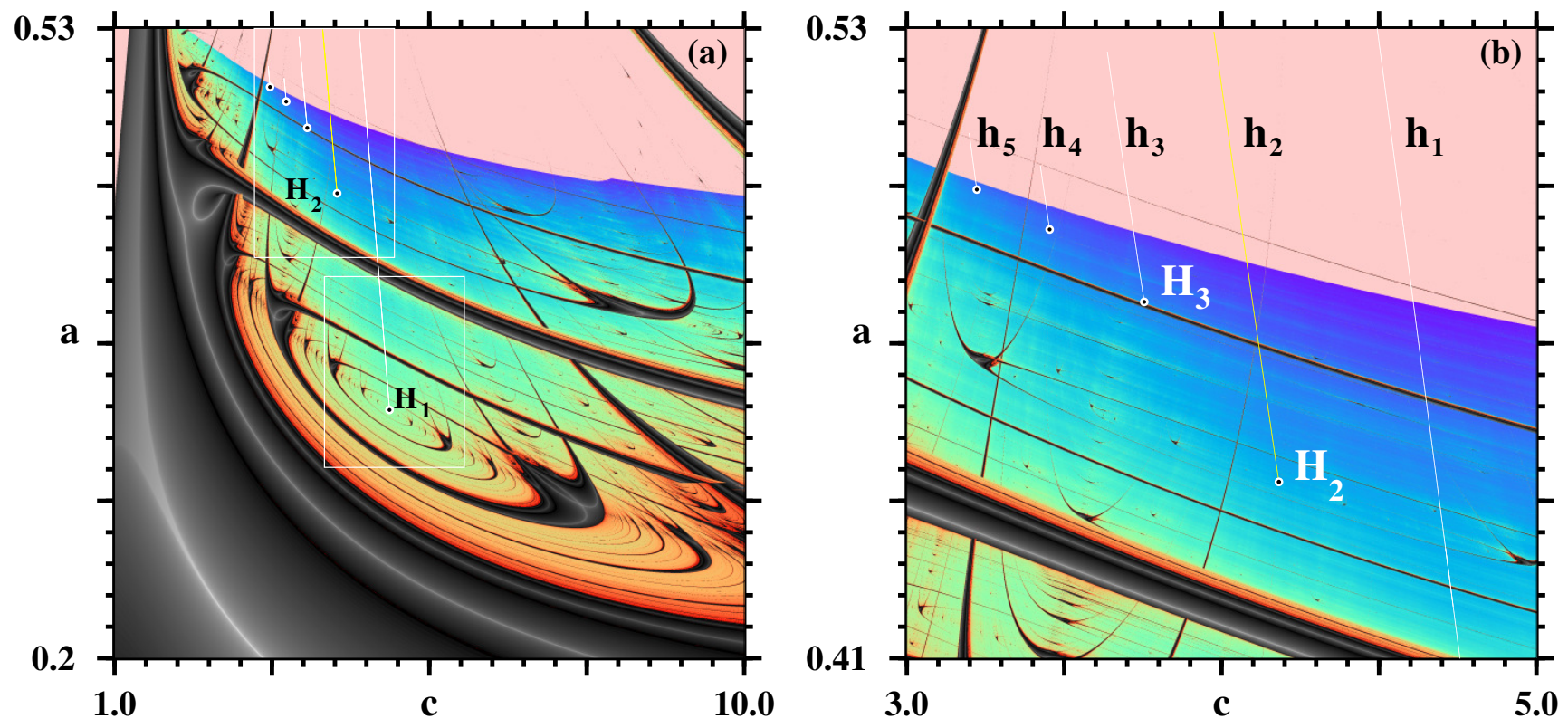

FIG. 2. (Color online) (a) Lyapunov phase diagram for Rössler's oscillator showing two periodicity hubs, $H_{1}$ and $H_{2}$, with their associated spirals and homoclinic loci. The box around $H_{1}$ is magnified in Fig. 1. (b) Magnification of box around $H_{2}$, also showing Shilnikov bifurcation curves $h_{i}, i=1, \ldots, 5$. Further magnifications would be required to visualize the self-similar spiral-like structures near $H_{k}, k \geq 3$. Parameter regions containing the letters (a) and (b) correspond to divergence of the orbits. Each panel displays phase-space analysis of $2400^{2}=5.76 \times 10^{6}$ individual parameter points.

icity spiral, the waveforms of the stable oscillations evolve continuously and their periodicity grows without bound. Also, sequences of shrimps [19-21] occur along the periodicity spiral arranged in consecutive pairs at each halfturn around $H_{1}$.

Similar spiral structures have been recently observed experimentally [17] in a slight variation of the same electric circuit where they were first anticipated by means of Lyapunov phase diagrams [3]. For applications, periodicity hubs are appealing because in their vicinity the control parameter space is organized into a predictable pattern of regular and chaotic regions. Hence, at a periodicity hub it is possible to selectively switch parameters between distinct periodicity spirals (characterized by periodic orbits with distinct waveforms and periods) without the need of ever crossing any of the surrounding chaoticity spirals and vice versa.

Initially, the research focused on an isolated periodicity hub [3]. In general, however, periodicity hubs are not isolated points but seem to always occur in infinite sequences (cascades), several of which can coexist, forming complex networks of self-similar structures in parameter space [6]. All the work so far has presented qualitative descriptions of the organization induced around periodicity hubs in the parameter space without explaining their origin. The aim of this paper is to remedy this situation by describing a global mechanism producing both 'isolated' cascades and large networks of periodicity hubs, responsible for organizing distinct overlapping sets of sta- ble oscillations in Lyapunov phase diagrams of dissipative flows. In particular we show how local results of Gaspard et al. [22, 23] (see also Ref. [24]) for Shilnikov homoclinic bifurcations can manifest themselves globally in the Lyapunov phase diagrams and comment on the additional detail that this interpretation allows us to deduce.

\section{GENESIS OF HUB CASCADES}

As a convenient working example, we consider again Rössler's oscillator, one of the most paradigmatic dissipative flows described in textbooks [18]. This model has the advantage of having many aspects of the local homoclinic phenomena arising from Shilnikov's scenario [25-30] as already described in early pioneering work by Gaspard et al. [22, 23] (see also Ref. [24]). Here we focus on global aspects connected with homoclinic phenomena and, more importantly, on their relation with the organization of networks of periodicity hubs in Lyapunov phase diagrams of dissipative flows. For terminology and general background material about homoclinic phenomena we refer to Refs. [2, 31, 32]. Without loss of generality we fix $b=0.3$, following Gaspard et al. [22, 23]. For $b=0.4$, the value considered by Fraser and Kapral [33], we observe the same global scenario which is the subject of this paper, see [6] for Lyapunov phase diagrams in that case.

As indicated by Figs. 1 and 2, periodicity hubs influ- 

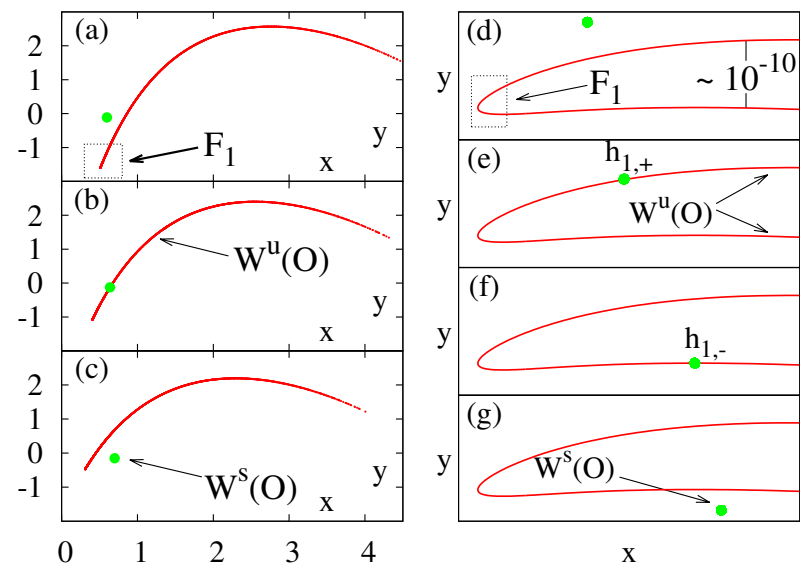

FIG. 3. (Color online) Illustration of homoclinic bifurcations in Rössler's oscillator. Left column: primary intersections of the manifolds $W^{s}(O)$ (dot) and $W^{u}(O)$ (curve) with the plane $\Sigma$ for $a=0.35$ fixed and: (a) $c=5.2$, (b) $c=4.88722732$, (c) $c=4.6$, showing the transit of $W^{s}(O)$ across $W^{u}(O)$ when the parameter $c$ is changed. Right column: sketches magnifying the folding region $F_{1}$ of the unstable manifold $W^{u}(O)$. (d) and (g): sketches of (a) and (c), respectively. What looks like a single curve in the left column panels is a pair of curves, which are narrowly spaced due to strong compression in the normal direction. Two distinct homoclinic bifurcations $h_{1,+}$ and $h_{1,-}$ take place for nearby parameter values, due to the folding and compression (panels $(\mathrm{e}, \mathrm{f})$ ). These two configurations cannot be distinguished from each other at the scale of panel (b).

ence extended portions of the parameter space. Figure 1 suggests that an infinite sequence of periodicity hubs $H_{1 j}$ converges to $H_{1}$ : most of the periodicity spirals attached to the $H_{1 j}$ can only be seen in magnifications of the Lyapunov phase diagram. However, a few shrimps along these periodicity spirals are visible at the scale of Fig. 1, where they are highlighted by circles. Additional hubs $H_{k}$ are revealed by examining a larger parameter domain (see Fig. 2(a)). The infinite alternation of spirals near $H_{2}$ is shown magnified in Fig. 2(b). By specialized numerical methods [32], we find several loci $h_{k}$ of Shilnikov homoclinic bifurcations [22-24] which, at the scale of Fig. 2 (b), seem to terminate at each hub $H_{k}$, for $k=1, \ldots, 5$.

We now address the fundamental question of this paper: what is the mechanism responsible for generating hubs and hub networks in Lyapunov phase diagrams and what is its relation with Shilnikov homoclinic bifurcations? A key concept here is that the hub network seen in parameter space is intimately related to the interaction in phase-space between the homoclinic orbits and the global structure of the Rössler attractor. Hence the discussion will now be focused on the structures in phase space.

To see how hub networks arise, we consider a subset of Rössler's $(a, c)$-parameter plane where the origin $O \equiv(0,0,0)$ is a saddle focus with a $1 \mathrm{D}$ stable manifold

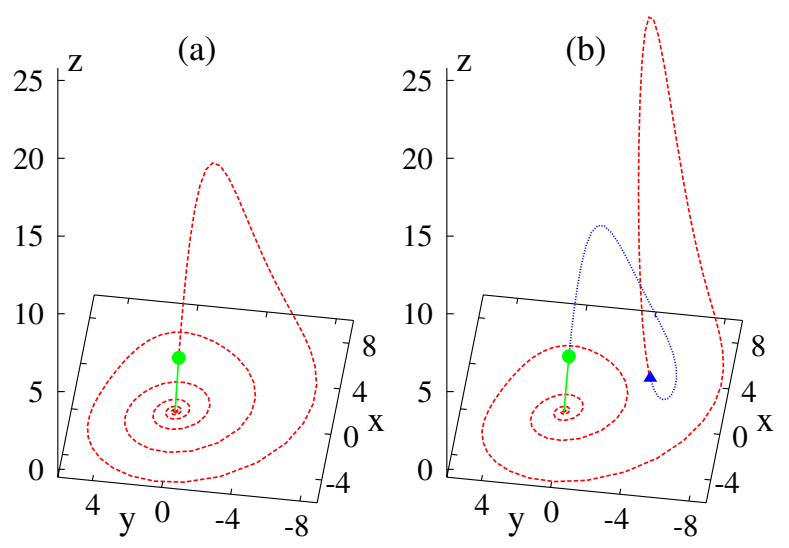

FIG. 4. (Color online) Homoclinic orbits in Rössler's oscillator, for parameter values at the periodicity hubs $H_{1}$ and $H_{2}$ as in Fig. 1. (a) A segment of $W^{s}(O)$ (green, solid) connects to an orbit segment in $W^{u}(O)$ (red, dashed) at a point on $\Sigma$ (green dot), for $(c, a)=(4.93029864,0.3301)$, corresponding to $H_{1}$. (b) A primary intersection point of $W^{u}(O)$ with $\Sigma$ (blue triangle) is mapped to the primary intersection point of $W^{s}(O)$ with $\Sigma$ (green dot) by the Poincaré map, for $(c, a)=(4.18195201,0.4436)$, corresponding to $\mathrm{H}_{2}$.

$W^{s}(O)$ and a 2D unstable manifold $W^{u}(O)$, see e.g. [22, Figure 1]. In this setting, the structure of the Rössler strange attractor is essentially determined by the geometry of $W^{u}(O)$. This geometry is analyzed as usual [2], in a Poincaré section of $W^{u}(O)$ by a surface $\Sigma$, taken here as the plane $z=3$ :

$$
\Sigma=\left\{(x, y, z) \in \mathbb{R}^{3} \mid z=3\right\} .
$$

As usual $[2,31,32]$, we examine the primary intersections of $W^{s}(O)$ and $W^{u}(O)$ with $\Sigma$ : loosely speaking, these consist of the points where orbits starting in the corresponding local manifolds hit $\Sigma$ for the first time [35].

The left column of Fig. 3 illustrates the process generating a homoclinic bifurcation, seen from within the Poincaré section $\Sigma$. The figure shows sections of the stable and unstable manifolds of the origin for three values of the parameter $c$, with $a$ fixed. For $c=5.2$ and $c=4.6$, the stable manifold $W^{S}(O)$ lies at different sides with respect to $W^{u}(O)$. The intermediate value of $c$ is chosen such that $W^{s}(O)$ actually enters $W^{u}(O)$ : this condition identifies a single point in the parameter plane for which a homoclinic bifurcation occur. Loosely speaking, to decrease the parameter $c$ with constant $a$ is equivalent to move $W^{s}(O)$ across $W^{u}(O)$. Figure 4 shows the homoclinic orbits occurring for parameter values $(c, a)$ at $H_{1}$ and $\mathrm{H}_{2}$ in Fig. 1.

The manifold $W^{u}(O)$ has a region of strong curvature where it folds onto itself. This region, labeled by $F_{1}$ in Fig. 3, is very hard to visualize in the original $(x, y)$ coordinates of $\Sigma$. This is due to strong compression in the normal direction to the unstable manifold $W^{u}(O)$, which in turn is due to the dissipativity of the system. To visu- 

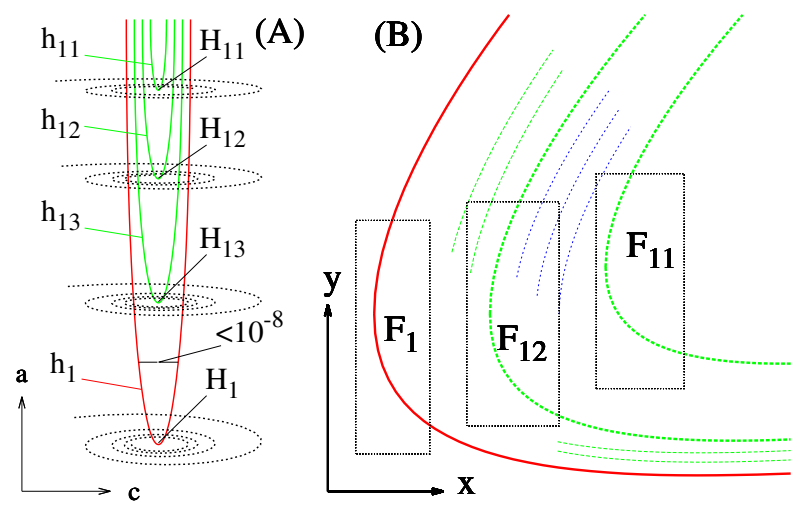

FIG. 5. (Color online) (A) Parameter plane sketch of the homoclinic loci $h_{1}, h_{11}, h_{12}, h_{13}$ (green, solid lines) with folding points at the hubs $H_{1}, H_{11}, H_{12}, H_{13}$. (B) Sketch within the Poincaré section $\Sigma$ of the primary (red, solid line) and secondary (green, dashed line) intersection of $W^{u}(O)$ with $\Sigma$, see [35], highlighting regions with strong curvature of $W^{u}(O)$, namely $F_{1}$ for the primary intersection and $F_{11}, F_{12}$ for the secondary intersection. Such regions of folding of the unstable manifold correspond to the hubs $H_{1}, H_{11}, F_{12}$ in panel (A).

alize the folding region we resort to hand-made sketches in the right panels of Fig. 3. The sketches clarify that a pair of homoclinic bifurcations $h_{1,+}$ and $h_{1,-}$ actually takes place near the folding region $F_{1}$. Since the width of the folding region is about $10^{-10}$, the two homoclinic bifurcations occur for values of the parameter $c$ which are very close to each other.

Having described how the homoclinic bifurcations occur for a fixed value of $a$, we now vary both parameters $(c, a)$ in order to locate the homoclinic loci $h_{i}$ in the parameter plane. These loci are obtained by tuning parameters so that $W^{s}(O)$ (the dot in the left column of Fig. 3) lies along $W^{u}(O)$ (the curve). We refer again to the sketches in the right column of Fig. 3: to decrease $a$ corresponds to shifting the pair of points $h_{1, \pm}$ towards the left (decreasing $x$ ). Hence, a pair of homoclinic bifurcation curves will occur in the parameter plane.

Again we see here the idea that structures in phase space have a close correspondence with structures in the parameter plane: the folding region of the unstable manifold $W^{u}(O)$ corresponds to a folding region for the homoclinic locus $h_{1}$ in Fig. 1. Hence, locus $h_{1}$ actually consists of a pair of narrowly spaced bifurcation curves, to which bifurcation points $h_{1,+}$ and $h_{1,-}$ of Fig. 3 (e,f) respectively belong. This pair of curves cannot be resolved at the scale of Fig. 1: as for Fig. 3, we must turn to a sketch in Fig. 5 (a) to illustrate the configuration of $h_{1}$. We now see that the periodicity hub $H_{1}$ occurs at the folding region of $h_{1}$ in Fig. 1.

It is worth to emphasize that, for a concrete system, the periodicity hub $H_{1}$ is not a single point in parameter space, rather a small region of strong curvature of the homoclinic locus $h_{1}$. Indeed, a single point is obtained only in the limit of infinite contraction for a local return map near the homoclinic orbit, see [23, Sec. 4]. However, contraction (due to dissipation) is usually so large that that periodicity hubs can be regarded as points for all practical purposes in many systems encountered in the applications.

Local analysis near the homoclinic orbit at the folding points reveals the basic structure of the periodicity spirals in a neighborhood of the hubs [23]. The standard theory of simple Shilnikov homoclinic bifurcations without folding points [24] shows that there is an infinite sequence of subsidiary homoclinic orbits accumulating on a principal homoclinic orbit. This yields an accumulation of homoclinic loci in parameter space from one side of the principal homoclinic locus. This theory can be adapted to the folding points to show that an infinite sequence of subsidiary folding points accumulates on a principal folding point with a geometrical rate. The position of the subsidiary foldings relative to the principal folding (i.e. outside or inside the principal folding point) depends on the sign of two normal form coefficients [36].

For the Rössler system (1), we find the subsidiary folding points to lie inside the principal folding at $F_{1}$, see Fig. 5 (b). The fourth largest periodicity hub $H_{11}$ in Fig. 1 is generated by a similar mechanism as $H_{1}$, with the difference that a subsidiary branch of $W^{u}(O)$ is involved - namely that giving rise to $F_{11}$ in Fig. 5 (b) - instead of the principal branch of Fig. 4 (a), involved in $H_{1}$. Accordingly, $H_{11}$ is the folding region of a homoclinic locus $h_{11}$, which is nested within the principal folded locus $h_{1}$. Analogous considerations imply that folded homoclinic loci $h_{1 j}$ emerge from hubs $H_{1 j}, j=2,3, \ldots$ and are nested into one another, see the sketch in Fig. 5 (a). The whole process repeats ad infinitum: Subsidiaries within subsidiaries will also show up nearby, giving rise to a sheaf of homoclinic loci nested within $h_{1}$ in Fig. 1. This sheaf is tightly packed, due to contraction in phase space: the distance between the two branches of $h_{1}$ is less than $10^{-8}$, see the sketch in Fig. 5 (a).

A very similar bifurcation structure was analyzed in detail by de Feo et al. $[15,16]$ for the Colpitts oscillator, by means of both numerical continuation of bifurcations of periodic orbits and direct integration. Our analysis in the next section, however, clarifies the link between the multi-branch structure of the attractor and the global organization of the hub network. We also link the normal form coefficients of the return map to the position of the subsidiary folding points [36]. More importantly, the arrangement reported in our Fig. 5 is quite different from what is described in Fig. 13 of de Feo et al. [15].

\section{LYAPUNOV PHASE DIAGRAMS AND HOMOCLINIC LOCI}

So what can we learn from the Lyapunov phase diagram? Is it possible to somehow guess the location of homoclinic loci from Lyapunov phase diagrams? Before proceeding, we invite the reader to try to use the knowl- 


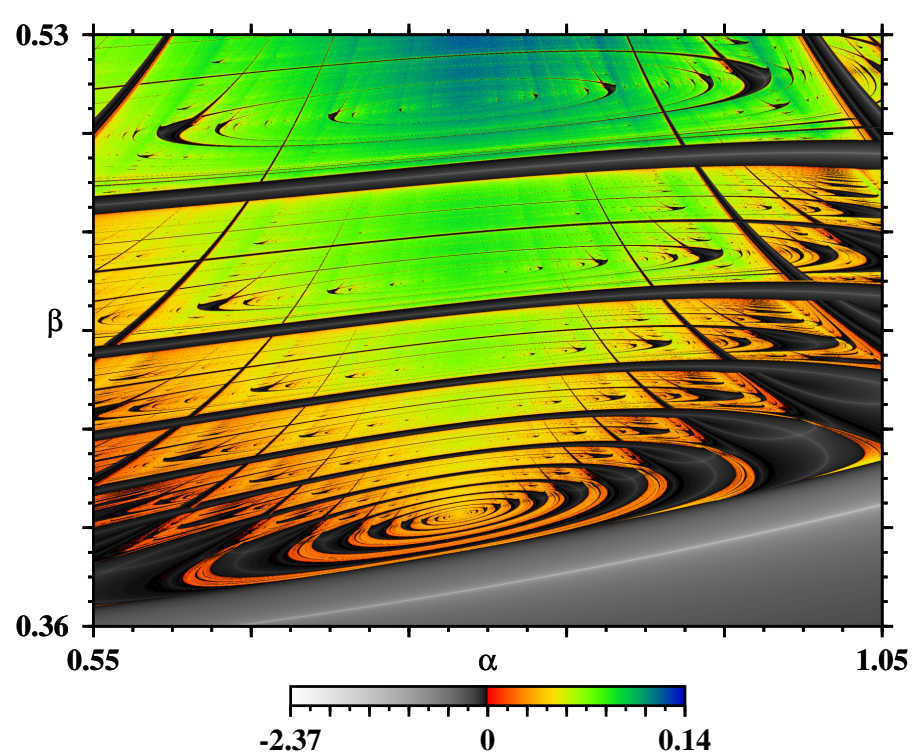

(a)

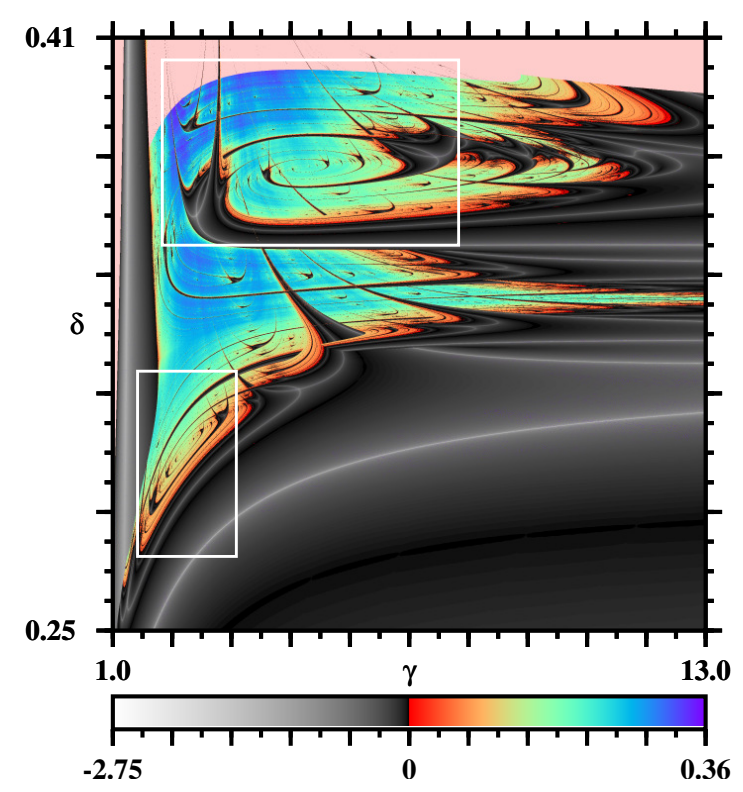

(b)

FIG. 6. (Color online) Two additional examples to illustrate the genericity of the global structure of hub networks. (a) hub network for a chemical oscillator $[6,22]$. (b) hub network in a simple electronic circuit with a tunnel diode [7]. White boxes mark the location of the two largest hub networks, many more exist. Lyapunov phase diagrams provide a quick guide about where to find homoclinic loci in control space of flows. Panels display $2400^{2}=5.76 \times 10^{6}$ parameter points.

edge won by reading the previous Section to guess where homoclinic loci are to be expect in the pair of Lyapunov phase diagrams shown in Fig. 6.

With hindsight, without resorting to computation of homoclinic bifurcations, the diagrams indeed show clearcut signatures which allow us to deduce the existence of homoclinic loci and locate their folding points. For example, the detection of the hub $H_{2}$ (Fig. 2) led us to try to explain this as part of the evolution of the Rössler attractor when changing parameters. The result of this investigation is shown in Fig. 7. Depending on the parameters, the intersection of the Rössler attractor (or, equivalently, of $W^{u}(O)$ ) with an appropriately chosen return plane develops well-separated bands. Each band has a fractal onion-like structure arising from the expanding, folding and return of the manifold $W^{u}(O)$ to the Poincaré section $\Sigma$. Also, each band has a point $F_{j}$ of strong curvature, where $W^{u}(O)$ is folded onto itself, just like $F_{1}$ in Fig. 3. According to Ref. [23], each folding contributes one periodicity hub through the mechanism illustrated in Figs. 3 and 5. Again, periodicity hubs centered at $\mathrm{H}_{2}$ and $\mathrm{H}_{3}$ in parameter plane (Fig. 2(b)) are induced by folding regions $F_{2}$ and $F_{3}$ of the unstable manifold in phase space (Fig. 7). Each periodicity hub $H_{k}$ is the folding point of a homoclinic locus $h_{k}$, with an associated sequence of subsidiary homoclinic loci $h_{k j}$ and their folding points $H_{k j}$. Since homoclinic loci have an infinite number of subsidiaries, we see nothing preventing the existence of an infinite number of such new independent hubs, as anticipated by numerical simulations [6].

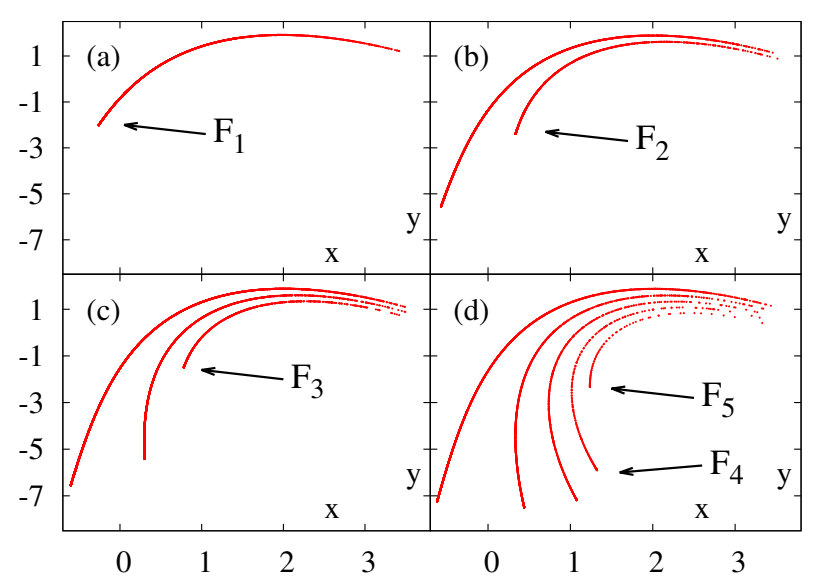

FIG. 7. (Color online) Intersection of $W^{u}(O)$ with the plane $\Sigma$ for $c=4$ and: (a) $a=0.4$, (b) $a=0.46$, (c) $a=0.475$, (d) $a=0.485$. Folding of $W^{u}(O)$ occurs near each $F_{j}$, compare with Fig. 3. Periodicity hubs arise at such foldings of the manifold, thus giving origin to an infinite network of hubs in control parameter space.

We emphasize that the existence of the extra folding points $F_{j}$, with $j>1$ is a novel and general feature that depends on specific details of the attractor of the system being considered: it does not follow from the local unfolding of a higher codimension bifurcation point in any obvious way (although there may still be a relationship with mixed-mode oscillation theory [37], see for exam- 
ple [38]). Correspondingly, the homoclinic orbits at the various hubs have a rather different structure (Fig. 4).

Seen in this light, periodicity hubs become a useful tool for the quick detection and understanding of global features of the parameter space. Features such as homoclinic folding points and their subsidiary bifurcations are normally only found by using specialized numerical methods, whilst a theoretical understanding of the Lyapunov phase diagrams allows the existence of these features to be deduced from the structure of the diagram itself.

The mechanism described here for the occurrence of periodicity hub networks involves Shilnikov homoclinic bifurcations. The latter are codimension-one phenomena, meaning that they are stably observed in systems depending on one parameter [2]. In the present scenario, individual cascades of periodicity hubs require at least two parameters, since a region of strong curvature is also needed along a curve of Shilnikov bifurcations. Hence, periodicity hubs can be considered as codimension-two phenomena, to be expected in systems with Shilnikov bifurcations when at least two parameters are varied. Technically speaking, a codimension-two condition is only obtained in the idealized situation of infinite contraction discussed above, see [23, Sec. 4].

Hub networks are indeed found in several physical models [6]: Fig. 6 shows Lyapunov phase diagrams for a chemical oscillator first introduced and analyzed by Gaspard et al. [6, 23] and for a circuit containing a tunnel diode [7], illustrating the similarity with the hub structure in Fig. 1. Hitherto, most work has concentrated on describing the features of the parameter space near the periodicity hubs found in these diagrams. Here we have complemented such understanding by showing how periodicity hubs are globally related to folding points of homoclinic loci described locally in Ref. [23], and have explained further properties of hubs close to a principal hub in terms of homoclinic bifurcation theory.

\section{CONCLUSIONS AND OUTLOOK}

In this paper we have described the global mechanism underlying the genesis and organization of networks of periodicity hubs in the control parameter space of a simple prototypical flow. We have clarified the relation between periodicity hubs and the presence of two distinct types of spiral-like regions, respectively characterized by families of periodic or chaotic oscillations. The link is made clear by Lyapunov phase diagrams, which give a fairly complete view of the dynamics and show the key role of periodicity hubs in organizing extended portions of the parameter space. Periodicity and chaoticity spirals in the parameter plane emerge from and accumulate at periodicity hubs: these are small regions of strong curvature of homoclinic bifurcation curves of a common saddle-focus equilibrium. These homoclinic bifurcation curves are arranged in fractal-like sheaves in the parameter plane.
The specific organization of hub networks was shown to depend strongly on the interaction between the homoclinic orbits and the global structure of the underlying attractor. Based on considerations of genericity in dynamical systems [2, 31], we argued that the mechanism described here, responsible for hub networks, might be found in a wide class of dissipative flows, namely a subset of those where Shilnikov's homoclinic scenario occurs $[2,18,22-31]$. At the same time, we do not exclude the possibility that periodicity hubs occur in systems with other non-Shilnikov reinjection mechanisms, see e.g. [5]. Furthermore, there exist examples of Shilnikov's scenario in the literature, without detectable periodicity hubs.

Lyapunov phase diagrams are efficient and useful exploratory tools for applications, to understand global features of complex attractors. For Rössler's system, the homoclinic locus $h_{2}$ was first computed using specialized numerical methods to detect homoclinic phenomena [22]. However, the existence of $h_{2}$ and the several additional homoclinic loci $h_{k}$ with folding points has been read off here directly from Lyapunov phase diagram like Fig. 2, a fact that greatly simplified their precise numerical calculation. This led to the discovery of the global banded structure of Rössler's attractor (Fig. 7) and of the additional hubs related to this structure. We believe that the use of Lyapunov phase diagrams can significantly augment and speed-up the understanding of physical models: such diagrams, focused on experimentally measurable features, reveal the occurrence of many global bifurcations without recourse to more specialized numerical techniques. They are therefore a very powerful way to begin the analysis of nonlinear systems and can also be applied to laboratory experiments which, of course, only detect stable structures. A complementary tool that may be useful in analyzing dynamical systems is the direct study of the oscillations as parameters are tuned [37].

In conclusion, although our emphasis here was on Shilnikov's homoclinic scenario, periodicity hubs have been reported very recently for a semiconductor laser with optoelectronic feedback [5], which is an excitable system with multiple time-scale dynamics [39, 40]. As anticipated theoretically by Marino et al. [39], such considerably richer scenarios are possible in higher-dimensional systems, particularly when period-doubling cascades follow a Hopf bifurcation and subsequent canard explosion, producing alternations of periodic and chaotic oscillations. As the amplitude of the chaotic attractors grows one observes a spiking regime consisting of large pulses separated by irregular time intervals in which the system displays small-amplitude chaotic oscillations. This scenario, reminiscent of Shilnikovs homoclinic chaos despite the fact that no homoclinic connections are involved, has already been observed very recently in ground-breaking experimental studies of a semiconductor laser [41, 42]. An interesting challenge now is to uncover the mechanism generating networks of hubs for such non-Shilnikov systems [5]. 
RV acknowledges support by the Willis Research Network. (www.willisresearchnetwork.com). JACG thanks the Royal Society for a fruitful month spent in Exeter, in June 2009. He also thanks support from the AFOSR Grant FA9550-07-1-0102, and CNPq, Brazil. Bitmaps were computed in the CESUP-UFRGS clusters.
[1] Y.C. Lai and T. Tél, Transient Chaos, (Springer, New York, 2011).

[2] P. Glendinning, Stability, Instability and Chaos, (Cambridge University Press, Cambridge, 1994).

[3] C. Bonatto and J.A.C. Gallas, Phys. Rev. Lett. 101, 054101 (2008).

[4] G.M. Ramírez-Ávila and J.A.C Gallas, Revista Boliviana de Fisica 14, 1-9 (2008); Phys. Lett. A 375, 143 (2010).

[5] J.G. Freire and J.A.C. Gallas, Phys. Rev. E 82, 037202 (2010).

[6] J.A.C. Gallas, Int. J. Bif. Chaos 20, 197 (2010).

[7] R.E. Francke and J.A.C. Gallas, Observation of spirals and infinite hub hierarchies in self-excited oscillations of a tunnel diode, preprint, 2011.

[8] H.W. Broer, C. Simó and R. Vitolo, Phys. D 237(13), 1773 (2008).

[9] R. Vitolo, C. Simó and H.W. Broer, Nonlinearity, 23, 1919 (2010).

[10] H.W. Broer, C. Simó and R. Vitolo, Discrete Contin. Dyn. Syst. B, 14(3) 871 (2010).

[11] A.E. Sterk, R. Vitolo, H.W. Broer, C. Simó, and H. Dijkstra. Phys. D, 239(10) 702 (2010).

[12] R. Vitolo, C. Simó and H.W. Broer, Regul. Chaotic Dyn. 16(1-2), 154 (2011).

[13] V. Kovanis, A. Gavrielides, and J.A.C. Gallas, Eur. Phys. J. D 58, 181 (2010).

[14] A. Celestino, C. Manchein, H.A. Albuquerque, and M.W. Beims, Phys. Rev. Lett. 106, xxx (2011), in print.

[15] O. de Feo, G.M. Maggio, and M.P. Kennedy, Int. J. Bif. Chaos 10, 935 (2000).

[16] O. de Feo and G.M. Maggio, Int. J. Bif. Chaos 13, 2917 (2003).

[17] R. Stoop, P. Benner, and Y. Uwate, Phys Rev. Lett. 105, $074102(2010)$.

[18] O.E. Rössler, Ann. N.Y. Acad. Sci. 316, 376 (1979).

[19] Shrimps are formed by a regular set of adjacent windows centered around a main pair of intersecting superstable parabolic arcs. A shrimp is a doubly infinite mosaic of stability domains composed by an innermost main domain plus all the adjacent stability domains arising from two period-doubling cascades together with their corresponding domains of chaos [20]. Shrimps should not be confused with their innermost main domain of periodicity.

[20] J.A.C. Gallas, Phys. Rev. Lett. 70, 2714 (1993); Physica A 202, 196 (1994); Appl. Phys. B 60, S203 (1995); B.R. Hunt, J.A.C. Gallas, C. Grebogi, J.A. Yorke and H. Koçak, Physica D 129, 35 (1999).

[21] E.N. Lorenz, Physica D 237, 1689 (2008).

[22] P. Gaspard and G. Nicolis, J. Stat. Phys. 31, 499 (1983). There is a misprint in Eq. (2) of this paper: in the last equation the term $-c x$ should be replaced by $-c z$.

[23] P. Gaspard, R. Kapral, G. Nicolis, J. Stat. Phys. 35, 697 (1984).

[24] P. Glendinning and C. Sparrow, J. Stat. Phys. 35, 645
(1984).

[25] L.P. Shilnikov, Sov. Math. Dokl. 6, 163 (1965); Sov. Math. Dokl. 8, 54 (1967); Math. SSSR Sbornik, 10, 91 (1970).

[26] N.K. Gavrilov and L.P. Shilnikov, Math SSSR Sbornik 19, 139 (1973). L.P. Shilnikov, A. Shilnikov, D. Turaev, and L. Chua, Methods of Qualitative Theory in Nonlinear Dynamics, (World Scientific, Singapore, 2001).

[27] L.P. Shilnikov and A. Shilnikov, Scholarpedia, 2(8), 1891 (2007).

[28] L.A. Belyakov, Math. Notes 15, 336 (1974); Math. Notes 28, 910 (1981); Math. Notes 36, 681 (1984).

[29] A. Arneodo, P. Coullet, and C. Tresser, Commun. Math. Phys. 79, 573 (1981); J. Stat. Phys. 27, 171 (1982).

[30] V.V. Bykov, Physica D 62, 290 (1993).

[31] M.W. Hirsch, S. Smale, and R.L. Devaney, Differential Equations, Dynamical Systems and An Introduction to Chaos, Chapter 16, (Elsevier, Amsterdam, 2004).

[32] C. Simó, On the analytical and numerical approximation of invariant manifolds. Les Méthodes Modernes de la Mécanique Céleste, D. Benest and C. Froeschlé, editors, (Editions Frontières, Paris, 1990), 285.

[33] S. Fraser and R. Kapral, Phys. Rev. A 25, 3223 (1982).

[34] C. Bonatto, J.C. Garreau, and J.A.C. Gallas, Phys. Rev. Lett. 95, 143905 (2005).

[35] The origin $O$ is always an equilibrium of Eqs. (1). The local stable and unstable manifold of $O$ are here represented as adapted parametrisations [32] restricted to a neighborhood of $O$ so that their image does not intersect $\Sigma$. Points on orbits starting in the local manifolds are naturally ordered by their integration time: primary, secondary and higher-order intersections with $\Sigma$ are defined by this ordering. For definiteness, we restrict to intersections where the flow is downward (upward) with respect to the $z$-axis for orbits in $W^{u}(O)\left(W^{s}(O)\right)$.

[36] The position of the subsidiary folding points depends on the constants $c$ and $d$ of the quadratic map $T_{1}$ modelling the global flow near the homoclinic orbit ([23], p. 711). If $c d>0$ then the subsidiary foldings are within the folding, whilst if $c d<0$ then they go around the folding on the outside. The choice of parameters used to model eqs. (1) has $c d>0$ ([23], p. 713).

[37] J.G. Freire and J.A.C. Gallas, Phys. Lett. A 375, 1097 (2011); Phys. Chem. Chem. Phys. 13, ??? (2011), in print.

[38] P. Gaspard and X.-J. Wang, J. Stat. Phys. 48, 151 (1987).

[39] F. Marino, F. Marin, S. Balle, and O. Piro, Phys. Rev. Lett. 98, 074104 (2007).

[40] F. Marino and F. Marin, Phys. Rev. E 83, 015202(R) (2011).

[41] K. Al-Naimee, F. Marino, M. Ciszak, R. Meucci and F.T. Arecchi, New J. Phys. 11, 073022 (2009).

[42] K. Al-Naimee, F. Marino, M. Ciszak, S.F. Abdalah, R. Meucci and F.T. Arecchi, Eur. Phys. J. D 58, 187 (2010). 